\title{
The role of tetrahydrobiopterin in inflammation and cardiovascular disease
}

\author{
Eileen McNeill ${ }^{1,2}$ and Keith M. Channon ${ }^{1,2}$ \\ ${ }^{1}$ Department of Cardiovascular Medicine, University of Oxford, John Radcliffe Hospital, Oxford, \\ UK \\ ${ }^{2}$ Wellcome Trust Centre for Human Genetics, University of Oxford, Oxford, UK
}

\section{Summary}

The cofactor tetrahydrobiopterin (BH4) is required for nitric oxide (NO) production by all nitric oxide synthase (NOS) enzymes and is a key regulator of cellular redox signalling. When BH4 levels become limiting NOS enzymes become 'uncoupled' and produce superoxide rather than NO. Endothelial cell BH4 is required for the maintenance of vascular function through NO production, and reduced BH4 levels are associated with vascular dysfunction. Evidence increasingly points to important roles for BH4 and NOS enzymes in other vascular cell types. Leukocytes have a fundamental role in atherosclerosis, and new evidence points to a role in the control of hypertension. Leukocytes are a major site of iNOS expression, and the regulation of this isoform is another mechanism by which $\mathrm{BH} 4$ availability may modulate disease. This review provides an overview of $\mathrm{BH} 4$ control of NOS function in both endothelial cells and leukocytes in the context of vascular disease and current therapeutic evaluations.

\section{Keywords}

Tetrahydrobiopterin; BH4; vascular disease; reactive oxygen species (ROS); nitric oxide

\section{Introduction}

Generation of nitric oxide (NO) by the vascular endothelium is a pivotal regulator of normal vascular homeostasis. Loss of normal NO-mediated endothelial function is an early and characteristic feature of vascular disease states, and is prospectively associated with adverse clinical risk in patients with vascular disease risk factors. Impaired endothelial function is also associated with increased production of reactive oxygen species (ROS), and reactive nitrogen species (RNS), in part through rapid reactions with NO, which reduce NO bioactivity. However, these reactions also mediate signalling through redox modification of

For personal or educational use only. No other uses without permission. All rights reserved.

Correspondence to: Prof. Keith Channon, Department of Cardiovascular Medicine, University of Oxford, John Radcliffe Hospital, Level 6 West Wing, Headley Way, Oxford, OX3 9DU, UK, Tel.: +44 1865572783, Fax: +44 1865287586,

Keith.Channon@cardiov.ox.ac.uk.

Conflicts of interest

None declared. 
target molecules that have important roles in both vascular physiology and disease pathogenesis. Vascular diseases are also associated with systemic inflammation, reflected for example in elevated levels of markers such as $\mathrm{C}$-reactive protein, and by direct involvement of inflammatory cells in vascular disease pathogenesis, where NO production is implicated in inflammatory cell function. Thus, NO has multiple and complex roles in both the pathophysiology of the vascular wall, and in the local and systemic inflammatory response.

The NO synthase (NOS) enzymes catalyse the formation of NO by oxidation of L-arginine, reduction of molecular oxygen and production of a by-product, L-citrulline. In NOS catalysis, $\mathrm{BH} 4$ controls 'coupling' of the haem-oxygen intermediate to L-arginine oxidation, thus controlling the generation of either NO or superoxide (-Fig. $1 \mathrm{~A})(1,2)$. When BH4 levels become deficient the net output of NOS enzymes becomes superoxide, produced from the oxygenase domain of the enzyme. The ability of the NOS enzymes to produce both NO, which maintains vascular function, and superoxide, underlies the critical importance of controlling NOS function in vascular disease. If NOS enzymes become uncoupled, the superoxide they produce can undergo immediate reactions to produce other ROS such as peroxynitrite or hydrogen peroxide, which can greatly alter the balance of redox signalling, leading to cellular injury and inflammation. The careful balance of NO/ROS production by NOS is intimately linked to cellular BH4 levels, and increased ROS production from uncoupled NOS enzymes can further limit BH4 availability through oxidation of this molecule. Once oxidised to BH2 (7,8-dihydrobiopterin), or other oxidised forms, BH4 is no longer able to maintain NOS coupling, propagating ROS production by the enzyme, that may in turn lead to further $\mathrm{BH} 4$ oxidation (3). Indeed, $\mathrm{BH} 2$ is reported to act as a competitive inhibitor at the $\mathrm{BH} 4$ binding site, making the ratio between $\mathrm{BH} 4$ and $\mathrm{BH} 2 \mathrm{a}$ determinant of NOS coupling $(4,5)$. Thus, BH4 regulation expands the range of NOS signalling in disease to include not only NO, but multiple ROS and NO-ROS effects that are important in vascular disease pathogenesis and mediate inflammation.

\section{Tetrahydrobiopterin - a redox sensor and effector}

BH4 is synthesised through a multistep pathway initiated by GTP cyclohydrolase I $(\mathrm{GTPCH})$ (-Fig. 1B) (6) . GTPCH, encoded by $\mathrm{GCH} 1$, is the rate-limiting enzyme in BH4 biosythesis $(5,7)$ and $G C H 1$ expression is the key determinant of endothelial cell BH4 levels and eNOS regulation in endothelial cells (8-11). Thus, understanding the stimuli that induce or modulate $\mathrm{GCH} 1$ expression is as important as understanding the expression of NOS isoforms themselves. eNOS is constitutively expressed in endothelial cells, but external factors such as laminar sheer stress upregulate eNOS expression (12). Laminar sheer stress also regulates BH4 levels, in this case rather than as a result of alterations in $G C H 1$ expression GTPCH protein undergoes phosphorylation, which enhances enzymatic activity (13). In diseased vessels eNOS levels can be reduced in endothelial cells overlying atherosclerotic plaque (14). However, in many conditions causing decreased vascular function, such as diabetes and hypertension, eNOS levels are not reduced $(15,16)$. In these conditions it is the availability of BH4 that drives effects via vascular dysfunction, rather than a lack of eNOS per se. 
GCH1 is upregulated by inflammatory cytokines, such as tumour necrosis factor-a, pathogen associated stimuli such as LPS (17), and oxidative stimuli such as hydrogen peroxide (18). More specifically cytokine stimuli have been shown to mediate $G C H 1$ transcription through the coordinate induction of the nuclear factor- $\mathrm{xB}$ and JAK-STAT pathway (19). These stimuli are potent inducers of GTPCH in multiple cell types in addition to endothelial cells (20). In particular macrophages are very sensitive to inflammatory and innate immune stimuli, increasing BH4 levels rapidly in response to these stimuli (21). Studies have shown that $G C H 1$ is induced even more widely within the immune system, being induced in T-cells by activation of the T-cell receptor and co-stimulatory signal (22). This modulation by inflammatory stimuli implicates BH4 in immunity and the response to inflammation more widely than just by maintaining vascular function. In addition to regulation via transcription GTPCH activity is also regulated by feedback inhibition, effected via GTPCH feedback regulatory protein (GFRP) and BH4 itself (23). BH4 levels can also be recovered by production of $\mathrm{BH} 4$ via a dihydrofolate reductase (DHFR)-mediated salvage pathway from the oxidised pterin BH2 ( $\bullet$ Fig. 1B) (24). Interestingly, regulation of other elements of the BH4 biosynthetic pathway by inflammatory stimuli has also been reported, such as the induction of PTPS in endothelial cells in response to interleukin (IL)-1beta (25) and the downregulation of GFRP in monocytic cells by LPS (26). GTPCH activity can also be modulated by phosphorylation, for example sheer stress causes phosphorylation on serine 81 via casein kinase II (13) and T-cell activation causes phosphorylation on serine 72 (22). Taken together these data show that the body has multiple methods of controlling BH4 levels in both health and in disease states, particularly in response to inflammatory stimuli, implying availability of $\mathrm{BH} 4$ is critical.

The control of cellular BH4 is important more widely in biology, with BH4 acting as a cofactor for aromatic amino acid hydoxylases, controlling epinephrine, norepinephrine, dopamine and 5-HT levels, and in ether lipid metabolism through regulation of AGMO activity $(27,28)$. The control of cellular BH4 levels are reviewed in more detail elsewhere (3).

\section{NOS enzymes in cardiovascular disease}

NOS enzymes exist in three forms: neuronal NOS (nNOS, NOS1); endothelial NOS (eNOS, NOS3) and inducible NOS (iNOS, NOS2). The nNOS isoform has important roles in the control of autonomic function in cardiovascular disease, which are outside the scope of this review (see [29] for an overview). Inducible NOS has profound effects on vascular function under conditions of sepsis, where it mediates profound hypotension, and is a potent mediator of inflammation (30). The eNOS isoform has a well-established role in vascular disease states such as atherosclerosis, hypertension, and ischaemia-reperfusion, which are all characterised by abnormalities in endothelial function. In endothelial cells, NO generated by eNOS modulates blood flow and pressure and has a number of important anti-atherogenic effects (31-33). Deficient NO-mediated endothelial function is characteristic of cardiovascular diseases, correlates with risk factor profile and is an independent predictor of adverse cardiac events $(34,35)$. Experimental studies in $\mathrm{eNOS}^{-/-}$mice demonstrate that eNOS has necessary and sufficient roles in vascular disease pathophysiology (36). The $\mathrm{eNOS}^{-/-}$mice demonstrate expected deficiencies in endothelial-derived relaxation to NOS- 
dependent stimuli, such as acetylcholine, in organ bath experiments and are hypertensive (37). When crossed with the hyperlipidaemic $\mathrm{ApoE}^{-/-}$mouse line a lack of eNOS expression increases both diet-induced atherosclerosis and injury-induced neointima formation (38-40). More recent studies have addressed the degree of NOS uncoupling seen in in vivo disease models. ApoE-/- mice have both a reduced production of NO, compared to matched wildtype mice, and an overproduction of NOS-derived ROS species, as demonstrated by their inhibition with L-NAME treatment (41). However, the net effect of all mediators produced by eNOS is anti-atherogenic, as eNOS double knockout mice show an increase in aortic VCAM expression and corresponding increase in leukocyte rolling and adhesion in the carotid artery (41). Despite this experimental basis, there is no evidence that primarily targeting NO-mediated endothelial function can alone reduce vascular disease progression or risk. Indeed, simple pharmacological NO generation, such as the use of nitrate drugs, have shown no prognostic clinical benefits and are associated with adverse consequences such as impaired vasomotor responses and increased vascular oxidative stress (42).

Due to the dual nature of NOS enzymes, simple knockout approaches may provide a misleading impression of how the molecule acts in disease. By removing the whole enzyme both the beneficial (NO) and potentially damaging (ROS) output is removed. Whilst these experiments have demonstrated the net positive effect of eNOS expression, the logical idea that eNOS overexpression would therefore be protective is not the case (43). Overexpression of eNOS causes an acceleration of atherosclerotic disease, accompanied by a skewing of NOS output towards an increase in superoxide production due a relative lack of BH4 due to the high levels of eNOS expression. This phenomenon is reversed by BH4 supplementation, which causes a decrease in atherosclerotic burden in eNOS transgenic/ApoE ${ }^{-/-}$mice (43). Whilst this data shows a positive effect of BH4 supplementation in a model of abundant eNOS uncoupling, it does not address effects of BH4 under more physiological disease conditions.

Experimental studies targeting BH4 directly, through supplementation or via the biosynthesis pathway, have yielded a picture of how this molecule can switch the enzyme between a pro- and an anti-atherosclerotic state. The most simple of these have shown positive effects of $\mathrm{BH} 4$ supplementation in reducing plaque size and leukocyte infiltration in the ApoE-/- model of atherosclerosis $(44,45)$. These positive effects are assumed to be mediated through effects on eNOS, as they closely mimic the effects of endothelial-specific GCH1 overexpression. Studies using this mouse model have shown that increasing endothelial cell $\mathrm{BH} 4$ is protective in models of diabetic vascular dysfunction and atherogenesis $(8,9)$. It has been shown that a reduction in BH4 levels, through use of the synthesis inhibitor DAHP, alters vascular function ex vivo causing relaxation of arterial rings to occur through hydrogen peroxide production, which was reversible by a BH4 analogue (46). The therapeutic capacity for 'improving' NOS function is clearly demonstrated, but these do not reveal the NOS enzymes that are modulated to achieve these effects.

\section{Leukocytes in vascular disease}

Alongside the importance of the endothelial cell in vascular health and disease, leukocytes have a critical role in vascular disease pathogenesis. One of the earliest steps in the initiation 
of atherosclerosis is the recruitment of monocyte macrophages to the vessel wall. These cells consume oxidised lipids by scavenger receptor-mediated uptake, forming fatty streak lesions composed of foam cells. These inflammatory macrophages are a source of ROS within the developing plaque and also secrete cytokine factors that cause further local inflammation and cellular activation. This potentiation of inflammation causes a further recruitment of other components of the immune system such as T-lymphocytes, and leads to the formation of a more advanced and complex plaque (47-49). The process of leukocyte recruitment to plaque prone areas is intimately linked to endothelial biology, with the activation of endothelial cells at sites of turbulent flow causing expression of adhesion molecules that facilitate leukocyte migration (50). The production of NO by endothelial cell eNOS can modulate this process by direct effects on endothelial cell adhesion molecule expression, helping to maintain a non-inflamed endothelium. Outside this well accepted role for leukocytes in vascular disease pathogenesis there is a developing view that leukocytes may play an unexpected role in hypertension. Data from animal studies shows that animals deficient in either $\mathrm{T}$ lymphocytes or myeloid cells have a blunted response to angiotensin II induced hypertension $(51,52)$. Whilst a role for leukocytes in the vascular remodelling and inflammatory response to hypertension is well accepted, this role in mediating the hypertensive response per se is relatively unexpected (53).

\section{iNOS in vascular disease}

The major role of $\mathrm{BH} 4$ in vascular disease is frequently considered to be in regulating eNOS function in the endothelial cell layer. This focus on endothelial cell biology overlooks important roles for $\mathrm{BH} 4$ in the control of other NOS isoforms, in particular iNOS, which are expressed by other cell types. In contrast to the vasculoprotective role of eNOS, iNOS expression is regarded as detrimental, in particular due to its role in septic shock. The biology of iNOS is complex, it clearly has fundamental roles in physiology such as in host defense against pathogens such as Listeria monocytogenes (54). Yet in complex acute pathologies such as septic shock this enzyme has powerful beneficial and detrimental effects related to cell types which express iNOS. Expression in leukocytes has been shown to be beneficial in models of non-sterile sepsis, principally caecal ligation and puncture (CLP) (55). In models of sterile sepsis, such as intravenous LPS injection, expression of iNOS by smooth muscle cells mediates the profound hypotension, which can ultimately cause death and as such iNOS knockout mice can show protection in this model (54).

Expression of iNOS in inflammatory cells is deleterious in atherosclerotic plaque progression and ischaemia-reperfusion injury. iNOS RNA and protein is reported in human atherosclerotic lesions (14), where its presence has been shown to co-localise with areas of lipid oxidation and protein nitration $(56,57)$. In experimental $\mathrm{ApoE}^{-/-}$models of atherosclerosis iNOS expression has been found to exacerbate disease, with iNOS ${ }^{-/-}$animals showing a decreased disease burden following high-fat feeding (58-61). iNOS is expressed by multiple cell types within atherosclerotic plaque, including both macrophages and smooth muscle cells (56), with data from bone marrow chimera showing that iNOS expression by both leukocytes and parachenymal cells is proatherogenic (62). Whether these pro-inflammatory effects of iNOS are due to NO production and whether iNOS uncoupling occurs in these conditions is not clear. 
Induction of iNOS in inflammatory cells is accompanied by induction of $G C H 1$, leading to increased BH4 synthesis that is required for iNOS activity and inflammatory responses (63). The pro-inflammatory role of iNOS has been ascribed to the high enzymatic output of NO, which can in turn react with ROS to produce RNS such as peroxynitrite. In the macrophage the pronounced expression of BH4 and iNOS is logical given the role of these phagocytic cells in limiting microbial infection. Studies have also shown that BH4 levels are increased in T lymphocytes following activation via the T-cell receptor and is accompanied by an induction of NOS-mediated NO and superoxide production (22). Indeed, despite coinduction of $\mathrm{GCH} 1$ and $\mathrm{BH} 4$ synthesis, iNOS may increase systemic oxidation of $\mathrm{BH} 4$ in disease states such as atherosclerosis, leading to a relative decrease in $\mathrm{BH} 4$, due to formation of dihydrobiotperin (BH2) and biopterin (B) that are unable to act as NOS cofactors (64). In atherosclerotic plaque, iNOS expression contributes to peroxynitrite mediated effects such as 3 -nitrotyrosine levels and lipid peroxidation following high-fat feeding $(59,64)$, and generates ROS in the atherosclerotic vasculature (62). Thus, the regulation of both iNOSdependent NO production and ROS production by BH4 may play pathological roles in vascular disease, which may be opposing to the effects of BH4 on eNOS in endothelial cells ( $\backsim$ Fig. 2). However, the relative contribution of coupled vs. uncoupled iNOS is not clearly established in vascular diseases. The net effect of coupling iNOS could be either detrimental, by enhancing the high output of NO, or beneficial by reducing ROS production or may indicate that other strategies that silence all iNOS functions may be required ( $\neg$ Fig. 2).

Isolated protein systems have shown the formation of the iNOS dimer, which is required for activity, is more BH4 dependent than other NOS isoforms (2). More recent studies have shown that iNOS becomes uncoupled by a lack of BH4 and that superoxide induced oxidative loss of BH4 causes ROS formation (65). Additionally, restriction of arginine availability is reported to cause increased ROS output by iNOS (66). Reduced arginine availability is a facet of inflammation and the dual NO and superoxide production by iNOS causes high levels of peroxynitrite formation, which is a potent mediator of the antimicrobial role of iNOS (67). This regulation of ROS production by an additional co-factor may explain studies showing a default production of both NO and ROS by iNOS. Studies have begun to assess the degree to which iNOS uncoupling is seem in physiological and cellular systems, with reduced BH4 causing increased iNOS-uncoupling in T-cells and in renal allografts associated with a BH4-inhibitable superoxide production $(22,68)$. This leaves open questions about the mechanism by which iNOS is detrimental in vascular disease, which need to be answered before rational therapeutic strategies targeting BH4 availability can be designed.

\section{$\mathrm{BH} 4$ regulation in human inflammation}

The discordant roles for BH4 in endothelial function vs. inflammation are illustrated in recent studies in patients with coronary artery disease (CAD) ( Table 1). Impaired endothelial function in CAD patients is associated with reduced BH4 levels in vascular tissue, but with increased systemic levels of BH4 in plasma (69), that correlate with markers of systemic inflammation, such as plasma hsCRP. Acute inflammatory stimulation impairs endothelial function but increases plasma BH4, in parallel with increased IL-6 (70), demonstrating that inflammation leads to opposing changes in systemic vs. endothelial BH4 
effects. $G C H 1$ genetic variants that reduce $G C H 1$ expression (71) render subjects unable to upregulate BH4 levels following systemic inflammation. These individuals demonstrate impaired endothelial function following an acute inflammatory challenge when compared to individuals expressing the wildtype allele (70). This data implies that increases in endothelial BH4 induced by inflammatory stimuli are required to maintain coupled eNOS function in the context of a more oxidising inflammatory environment.

\section{$\mathrm{BH} 4$ as a therapeutic target}

Defining the relative roles of the different NOS isoforms and their signalling modalities is crucial for understanding both the utility and the limitations of BH4 'therapy' in vascular disease. Many clinical studies ( Table 1) have achieved positive effects on vascular function through local infusion of high-dose $\mathrm{BH} 4$ in patients with vascular dysfunction relating to diabetes, hypertension, coronary artery disease, hypercholesterolemia or cigarette smoking (72-76). These studies indicate that endothelial function in human subjects can be augmented by exogenous BH4, in the short term. However, long-term oral supplementation of BH4 has raised challenges that limit the potential of BH4 as a therapy in vascular disease. In younger subjects with hypercholesterolaemia, oral BH4 treatment improved endothelial function (77). In contrast, a double-blind placebo-controlled randomised clinical trial of oral BH4 in CAD patients prior to CABG surgery found no benefit of BH4 on endothelial function, with only a modest increases in vascular tissue BH4 levels, accompanied by an equal or greater increase in tissue $\mathrm{BH} 2$ (78). These observations suggest that increasing systemic levels of BH4 in subjects with advanced vascular disease and systemic inflammation is limited by rapid BH4 oxidation. Indeed, there is the possibility that systemic BH4 treatment may contribute to inflammation, rather than inhibiting the inflammation that leads to BH4 oxidation and endothelial dysfunction, through increasing iNOS activity. The simplistic notion that systemic BH4 supplementation will have net effects that are clinically beneficial cannot be presumed in cardiovascular disease states with different mechanisms at different stages of the disease progression (79).

There remains a pressing need for better understanding of the mechanistic role of the NOS enzymes and their regulation by BH4 in cardiovascular disease pathophysiology. The simplistic paradigms of increasing or inhibiting NOS activity, using unselective systemic approaches, must be refined by a more rational approach. Where previously the focus of much research and pre-clinical testing has been aimed purely at altering endothelial BH4 levels and 'improving' eNOS function, and thus reducing vascular dysfunction, it now seems clear that BH4 may have potent effects on other cells implicated in cardiovascular disease. How to formulate an agent to supplement BH4 in patients is an additional challenge, given the rapid oxidation of this molecule seem in the recent clinical study (78). If BH4 has competing or non-complementary roles in different cell types the next rational target may be to target the regulation of GTPCH within the target cell population to allow better specificity of effect to be achieved.

The increasing evidence that $\mathrm{BH} 4$ plays a role in regulating the function of multiple immune cell types opens up an exciting new field, particularly as this coincides with the recognition that conditions such as hypertension may have a strong immune component. It may be that 
existing strategies and technologies designed with endothelial BH4 and NOS biology in mind allow rapid progress in both defining the role of BH4 in inflammatory and immune processes and in harnessing this pathway for therapeutic benefit. However, there are still fundamental questions that need to be answered to allow translation of recent discoveries to patients. There are crucial differences in how humans and mice utilise the iNOS pathway, with this being a major player in murine innate immunity and macrophage function, as illustrated by the dependence on iNOS for survival from mycobacterial disease, yet in the human the role of iNOS in mTB infected macrophages is unclear (54). Similarly the clear role of iNOS in mediating and modulating sepsis in animal models, has not translated to human benefit. These early disappointments leave questions as yet unanswered about the contribution of iNOS (and indeed eNOS expressed by non-endothelial cells) to human pathology. Even when we consider the biology of BH4 in human leukocytes there are complications that need to be analysed such as the reduced capacity of human macrophages to produce BH4 (80). To move forward we need to understand how eNOS and iNOS enzymatic regulation and NO-ROS signalling, in both endothelial cells and inflammatory cells, are altered in cardiovascular disease states, and which of these mechanistic steps will provide realistic targets for therapy.

\section{Acknowledgements}

This work was supported by the British Heart Foundation [RG/10/15/28578 and RG/07/003/23133], the Wellcome Trust [090532/Z/09/Z] and the National Institute for Health Reseach (NIHR) Oxford Biomedical Research Centre. The authors would like to thank Dr Mark Crabtree for helpful comment on the manuscript.

\section{References}

1. Alp NJ, Channon KM. Regulation of endothelial nitric oxide synthase by tetrahydrobiopterin in vascular disease. Arterioscler Thromb Vasc Biol. 2004; 24:413-420. [PubMed: 14656731]

2. Stuehr DJ. Mammalian nitric oxide synthases. Biochim Biophys Acta. 1999; 1411:217-230. [PubMed: 10320659]

3. Crabtree MJ, Channon KM. Synthesis and recycling of tetrahydrobiopterin in endothelial function and vascular disease. Nitric Oxide. 2011; 25:81-88. [PubMed: 21550412]

4. Vasquez-Vivar J, et al. Superoxide generation by endothelial nitric oxide synthase: the influence of cofactors. Proc Natl Acad Sci USA. 1998; 95:9220-9225. [PubMed: 9689061]

5. Crabtree MJ, et al. Quantitative regulation of intracellular endothelial nitric oxide synthase (eNOS) coupling by both tetrahydrobiopterin-eNOS stoichiometry and biopterin redox status: Insights from cells with tet-regulated GTP cyclohydrolase I expression. J Biol Chem. 2009; 284:1136-1144. [PubMed: 19011239]

6. Werner-Felmayer G, et al. Tetrahydrobiopterin biosynthesis, utilization and pharmacological effects. Curr Drug Metab. 2002; 3:159-173. [PubMed: 12003348]

7. Tatham AL, et al. GTP cyclohydrolase I expression, protein, and activity determine intracellular tetrahydrobiopterin levels, independent of GTP cyclohydrolase feedback regulatory protein expression. J Biol Chem. 2009; 284:13660-13668. [PubMed: 19286659]

8. Alp NJ, et al. Tetrahydrobiopterin-dependent preservation of nitric oxide-mediated endothelial function in diabetes by targeted transgenic GTP-cyclohydrolase I overexpression. J Clin Invest. 2003; 112:725-735. [PubMed: 12952921]

9. Alp NJ, et al. Increased endothelial tetrahydrobiopterin synthesis by targeted transgenic GTPcyclohydrolase I overexpression reduces endothelial dysfunction and atherosclerosis in ApoEknockout mice. Arterioscler Thromb Vasc Biol. 2004; 24:445-450. [PubMed: 14707037]

10. Khoo JP, et al. A pivotal role for tetrahydrobiopterin in pulmonary hypertension. Circulation. 2005; 111:2126-2133. [PubMed: 15824200] 
11. Bendall JK, et al. Stoichiometric relationships between endothelial tetrahydrobiopterin, eNOS activity and eNOS coupling in vivo: Insights from transgenic mice with endothelial-targeted GTPCH and eNOS over-expression. Circ Res. 2005; 97:864-871. [PubMed: 16179591]

12. Traub O, Berk BC. Laminar shear stress: mechanisms by which endothelial cells can transduce an atheroprotective force. Arterioscler Thromb Vasc Biol. 1998; 18:677-685. [PubMed: 9598824]

13. Widder JD, et al. Regulation of Tetrahydrobiopterin Biosynthesis by Shear Stress. Circ Res. 2007; 108:830-838.

14. Wilcox JN, et al. Expression of multiple isoforms of nitric oxide synthase in normal and atherosclerotic vessels. Arterioscler Thromb Vasc Biol. 1997; 17:2479-2488. [PubMed: 9409218]

15. Cosentino F, et al. High glucose increases nitric oxide synthase expression and superoxide anion generation in human aortic endothelial cells. Circulation. 1997; 96:25-28. [PubMed: 9236411]

16. Bouloumie A, et al. Endothelial dysfunction coincides with an enhanced nitric oxide synthase expression and superoxide anion production. Hypertension. 1997; 30:934-941. [PubMed: 9336396]

17. Katusic ZS, et al. Cytokines stimulate GTP cyclohydrolase I gene expression in cultured human umbilical vein endothelial cells. Arterioscler Thromb Vasc Biol. 1998; 18:27-32. [PubMed: 9445252]

18. Shimizu S, et al. Hydrogen peroxide stimulates tetrahydrobiopterin synthesis through the induction of GTP-cyclohydrolase I and increases nitric oxide synthase activity in vascular endothelial cells. Free Radic Biol Med. 2003; 34:1343-1352. [PubMed: 12726922]

19. Huang A, et al. Cytokine-stimulated GTP cyclohydrolase I expression in endothelial cells requires coordinated activation of nuclear factor-kappaB and Stat1/Stat3. Circ Res. 2005; 96:164-171. [PubMed: 15604419]

20. Gross SS, Levi R. Tetrahydrobiopterin synthesis: an absolute requirement for cytokine-induced nitric oxide generation by vascular smooth muscle. J Biol Chem. 1992; 267:25722-25729. [PubMed: 1281471]

21. Sakai N, et al. Tetrahydrobiopterin is required for cytokine-induced nitric oxide production in a murine macrophage cell line (RAW 264). Mol Pharmacol. 1993; 43:6-10. [PubMed: 7678692]

22. Chen W, et al. Role of increased guanosine triphosphate cyclohydrolase-1 expression and tetrahydrobiopterin levels upon T cell activation. J Biol Chem. 2011; 286:13846-13851. [PubMed: 21343293]

23. Milstien S, et al. Purification and cloning of the GTP cyclohydrolase I feedback regulatory protein, GFR. J Biol Chem. 1996; 271:19743-19751. [PubMed: 8702680]

24. Curtius HC, et al. Tetrahydrobiopterin biosynthesis. Studies with specifically labeled $(2 \mathrm{H}) \mathrm{NAD}(\mathrm{P}) \mathrm{H}$ and $2 \mathrm{H} 2 \mathrm{O}$ and of the enzymes involved. Eur J Biochem. 1985; 148:413-419. [PubMed: 3888618]

25. Franscini N, et al. Critical role of interleukin-1beta for transcriptional regulation of endothelial 6pyruvoyltetrahydropterin synthase. Arterioscler Thromb Vasc Biol. 2003; 23:e50-53. [PubMed: 14551150]

26. Werner ER, et al. Bacterial lipopolysaccharide down-regulates expression of GTP cyclohydrolase I feedback regulatory protein. J Biol Chem. 2002; 277:10129-10133. [PubMed: 11799107]

27. Nagatsu T, et al. Udenfriend, Conversion of L-tyrosine to 3,4-dihydroxyphenylalanine by cell-free preparations of brain and sympathetically innervated tissues. Biochem Biophys Res Commun. 1964; 14:543-549. [PubMed: 5836553]

28. Kaufman S. The Structure of the Phenylalanine-Hydroxylation Cofactor. Proc Natl Acad Sci USA. 1963; 50:1085-1093. [PubMed: 14096182]

29. Chowdhary S, Townend JN. Role of nitric oxide in the regulation of cardiovascular autonomic control. Clin Sci. 1999; 97:5-17. [PubMed: 10369789]

30. Fortin CF, et al. Sepsis, leukocytes, and nitric oxide (NO): an intricate affair. Shock. 2010; 33:344352. [PubMed: 19789465]

31. Ignarro LJ. Nitric oxide as a unique signaling molecule in the vascular system: a historical overview. J Physiol Pharmacol. 2002; 53:503-514. [PubMed: 12512688]

32. Aicher A, et al. Essential role of endothelial nitric oxide synthase for mobilization of stem and progenitor cells. Nat Med. 2003; 9:1370-1376. [PubMed: 14556003] 
33. Landmesser U, et al. Statin-induced improvement of endothelial progenitor cell mobilization, myocardial neovascularization, left ventricular function, and survival after experimental myocardial infarction requires endothelial nitric oxide synthase. Circulation. 2004; 110:19331939. [PubMed: 15466656]

34. Cai H, Harrison DG. Endothelial dysfunction in cardiovascular diseases: the role of oxidant stress. Circ Res. 2000; 87:840-844. [PubMed: 11073878]

35. Hill JM, et al. Circulating endothelial progenitor cells, vascular function, and cardiovascular risk. N Engl J Med. 2003; 348:593-600. [PubMed: 12584367]

36. Liu VW, Huang PL. Cardiovascular roles of nitric oxide: a review of insights from nitric oxide synthase gene disrupted mice. Cardiovasc Res. 2008; 77:19-29. [PubMed: 17658499]

37. Huang PL, et al. Hypertension in mice lacking the gene for endothelial nitric oxide synthase. Nature. 1995; 377:239-242. [PubMed: 7545787]

38. Moroi M, et al. Interaction of genetic deficiency of endothelial nitric oxide, gender, and pregnancy in vascular response to injury in mice. J Clin Invest. 1998; 101:1225-1232. [PubMed: 9502763]

39. Chen J, et al. Hypertension does not account for the accelerated atherosclerosis and development of aneurysms in male apolipoprotein e/endothelial nitric oxide synthase double knockout mice. Circulation. 2001; 104:2391-2394. [PubMed: 11705813]

40. Kuhlencordt PJ, et al. Accelerated atherosclerosis, aortic aneurysm formation, and ischemic heart disease in apolipoprotein E/endothelial nitric oxide synthase double-knockout mice. Circulation. 2001; 104:448-454. [PubMed: 11468208]

41. Ponnuswamy $\mathrm{P}$, et al. eNOS protects from atherosclerosis despite relevant superoxide production by the enzyme in apoE mice. PloS one. 2012; 7:e30193. [PubMed: 22291917]

42. Schulz E, et al. Functional and biochemical analysis of endothelial (dys)function and NO/cGMP signaling in human blood vessels with and without nitroglycerin pretreatment. Circulation. 2002; 105:1170-1175. [PubMed: 11889009]

43. Ozaki M, et al. Overexpression of endothelial nitric oxide synthase accelerates atherosclerotic lesion formation in apoE-deficient mice. J Clin Invest. 2002; 110:331-340. [PubMed: 12163452]

44. Schmidt TS, et al. Tetrahydrobiopterin supplementation reduces atherosclerosis and vascular inflammation in apolipoprotein E-knockout mice. Clin Sci. 2010; 119:131-142. [PubMed: 20337596]

45. Hattori Y, et al. Oral administration of tetrahydrobiopterin slows the progression of atherosclerosis in apolipoprotein E-knockout mice. Arterioscler Thromb Vasc Biol. 2007; 27:865-870. [PubMed: 17272747]

46. Cosentino F, Katusic ZS. Tetrahydrobiopterin and dysfunction of endothelial nitric oxide synthase in coronary arteries. Circulation. 1995; 91:139-144. [PubMed: 7528647]

47. McNeill E, et al. Greaves, Inflammatory cell recruitment in cardiovascular disease: murine models and potential clinical applications. Clinical Sci. 2010; 118:641-655. [PubMed: 20210786]

48. Weber $\mathrm{C}$, et al. The multifaceted contributions of leukocyte subsets to atherosclerosis: lessons from mouse models. Nat Rev Immunol. 2008; 8:802-815. [PubMed: 18825131]

49. Libby P, et al. Inflammation and atherosclerosis. Circulation. 2002; 105:1135-1143. [PubMed: $11877368]$

50. Nakashima Y, et al. Upregulation of VCAM-1 and ICAM-1 at atherosclerosisprone sites on the endothelium in the ApoE-deficient mouse. Arterioscler Thromb Vasc Biol. 1998; 18:842-851. [PubMed: 9598845]

51. Guzik TJ, et al. Role of the T cell in the genesis of angiotensin II induced hypertension and vascular dysfunction. J Ex Med. 2007; 204:2449-2460.

52. Wenzel P, et al. Lysozyme M-positive monocytes mediate angiotensin II-induced arterial hypertension and vascular dysfunction. Circulation. 2011; 124:1370-1381. [PubMed: 21875910]

53. Harrison DG, et al. Inflammation, immunity, and hypertension. Hypertension. 2011; 57:132-140. [PubMed: 21149826]

54. MacMicking JD, et al. Altered responses to bacterial infection and endotoxic shock in mice lacking inducible nitric oxide synthase. Cell. 1995; 81:641-650. [PubMed: 7538909] 
55. Cobb JP, et al. Inducible nitric oxide synthase (iNOS) gene deficiency increases the mortality of sepsis in mice. Surgery. 1999; 126:438-442. [PubMed: 10455918]

56. Luoma JS, Yla-Herttuala S. Expression of inducible nitric oxide synthase in macrophages and smooth muscle cells in various types of human atherosclerotic lesions. Virchows Arch. 1999; 434:561-568. [PubMed: 10394893]

57. Cromheeke KM, et al. Inducible nitric oxide synthase colocalizes with signs of lipid oxidation/ peroxidation in human atherosclerotic plaques. Cardiovasc Res. 1999; 43:744-754. [PubMed: 10690346]

58. Detmers PA, et al. Deficiency in inducible nitric oxide synthase results in reduced atherosclerosis in apolipoprotein E-deficient mice. J Immunol. 2000; 165:3430-3435. [PubMed: 10975863]

59. Kuhlencordt PJ, et al. Genetic deficiency of inducible nitric oxide synthase reduces atherosclerosis and lowers plasma lipid peroxides in apolipoprotein E-knockout mice. Circulation. 2001; 103:3099-3104. [PubMed: 11425775]

60. Chen J, et al. Effects of chronic treatment with L-arginine on atherosclerosis in apoE knockout and apoE/inducible NO synthase double-knockout mice. Arterioscler Thromb Vasc Biol. 2003; 23:97103. [PubMed: 12524231]

61. Miyoshi T, et al. Deficiency of inducible NO synthase reduces advanced but not early atherosclerosis in apolipoprotein E-deficient mice. Life Sci. 2006; 79:525-531. [PubMed: $16516241]$

62. Ponnuswamy P, et al. Oxidative stress and compartment of gene expression determine proatherosclerotic effects of inducible nitric oxide synthase. Am J Pathol. 2009; 174:2400-2410. [PubMed: 19465644]

63. Hattori Y, et al. Tetrahydrobiopterin and GTP cyclohydrolase I in a rat model of endotoxic shock: relation to nitric oxide synthesis. Exp Physiol. 1996; 81:665-671. [PubMed: 8853274]

64. Upmacis RK, et al. Profound biopterin oxidation and protein tyrosine nitration in tissues of ApoEnull mice on an atherogenic diet: contribution of inducible nitric oxide synthase. Am J Physiol Heart Circ Physiol. 2007; 293:H2878-2887. [PubMed: 17766468]

65 . Sun J, et al. Reactive oxygen and nitrogen species regulate inducible nitric oxide synthase function shifting the balance of nitric oxide and superoxide production. Arch Biochem Biophys. 2010; 494:130-137. [PubMed: 19932078]

66. Xia Y, et al. Inducible nitric-oxide synthase generates superoxide from the reductase domain. $\mathbf{J}$ Biol Chem. 1998; 273:22635-22639. [PubMed: 9712892]

67. Linares E, et al. Role of peroxynitrite in macrophage microbicidal mechanisms in vivo revealed by protein nitration and hydroxylation. Free Radic Biol Med. 2001; 30:1234-1242. [PubMed: 11368921]

68. Huisman A, et al. Anti-inflammatory effects of tetrahydrobiopterin on early rejection in renal allografts: modulation of inducible nitric oxide synthase. FASEB J. 2002; 16:1135-1137. [PubMed: 12039851]

69. Antoniades C, et al. Altered plasma versus vascular biopterins in human atherosclerosis reveal relationships between endothelial nitric oxide synthase coupling, endothelial function, and inflammation. Circulation. 2007; 116:2851-2859. [PubMed: 18040031]

70. Antoniades C, et al. Induction of vascular GTP-cyclohydrolase I and endogenous tetrahydrobiopterin synthesis protect against inflammation-induced endothelial dysfunction in human atherosclerosis. Circulation. 2011; 124:1860-1870. [PubMed: 21969008]

71. Antoniades $\mathrm{C}$, et al. GCH1 haplotype determines vascular and plasma biopterin availability in coronary artery disease effects on vascular superoxide production and endothelial function. $\mathrm{J}$ Am Coll Cardiol. 2008; 52:158-165. [PubMed: 18598896]

72. Heitzer T, et al. Tetrahydrobiopterin improves endothelium-dependent vasodilation by increasing nitric oxide activity in patients with Type II diabetes mellitus. Diabetologia. 2000; 43:1435-1438. [PubMed: 11126415]

73. Higashi Y, et al. Tetrahydrobiopterin enhances forearm vascular response to acetylcholine in both normotensive and hypertensive individuals. Am J Hypertens. 2002; 15:326-332. [PubMed: 11991218] 
74. Stroes E, et al. Tetrahydrobiopterin restores endothelial function in hypercholesterolemia. J Clin Invest. 1997; 99:41-46. [PubMed: 9011574]

75. Heitzer T, et al. Tetrahydrobiopterin improves endothelium-dependent vasodilation in chronic smokers : evidence for a dysfunctional nitric oxide synthase. Circ Res. 2000; 86:E36-E41. [PubMed: 10666424]

76. Maier W, et al. Tetrahydrobiopterin improves endothelial function in patients with coronary artery disease. J Cardiovasc Pharmacol. 2000; 35:173-178. [PubMed: 10672847]

77. Cosentino F, et al. Chronic treatment with tetrahydrobiopterin reverses endothelial dysfunction and oxidative stress in hypercholesterolaemia. Heart. 2008; 94:487-492. [PubMed: 17916662]

78. Cunnington $\mathrm{C}$, et al. Systemic and vascular oxidation limits the efficacy of oral tetrahydrobiopterin treatment in patients with coronary artery disease. Circulation. 2012; 125:1356-1366. [PubMed: 22315282]

79. Cunnington C, Channon KM. Tetrahydrobiopterin: pleiotropic roles in cardiovascular pathophysiology. Heart. 2010; 96:1872-1877. [PubMed: 20837663]

80. Leitner KL, et al. Low tetrahydrobiopterin biosynthetic capacity of human monocytes is caused by exon skipping in 6-pyruvoyl tetrahydropterin synthase. Bio-chem J. 2003; 373:681-688. 


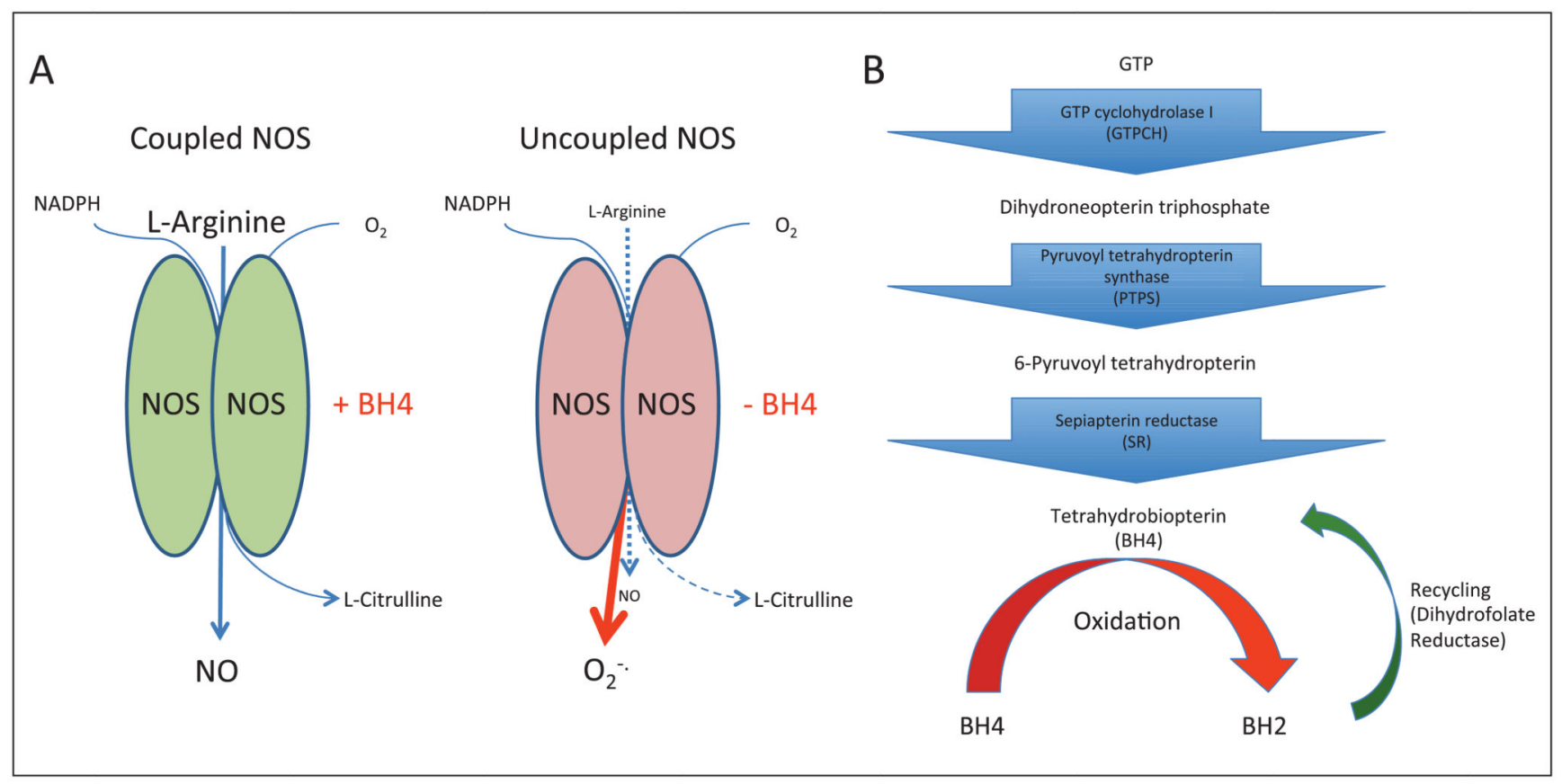

Figure 1. The biochemistry of NOS function and BH4 biosynthesis.

A) When BH4 levels are sufficient oxidation of L-arginine is coupled with the reduction of molecular oxygen to form NO and L-citrulline. When BH4 levels become limiting, due to either a reduced biosynthesis or due to oxidative loss, NOS enzymes become uncoupled and superoxide is produced as an alternative product of the enzyme. This production of superoxide can cause a further oxidative loss of BH4 potentiating a state of oxidative stress in the vasculature. B) BH4 is produced from GTP by a multi-step enzyme pathway. GTPCH is the rate-limiting enzyme in the majority of cells in the de novo production of $\mathrm{BH} 4$. $\mathrm{BH} 4$ is susceptible to oxidation, due to production of ROS from uncoupled eNOS or other ROS producing enzymes. $\mathrm{BH} 4$ can be reformed from the oxidised form, $\mathrm{BH} 2$, by the action of a salvage pathway, dependent on dihydrofolate reductase. 


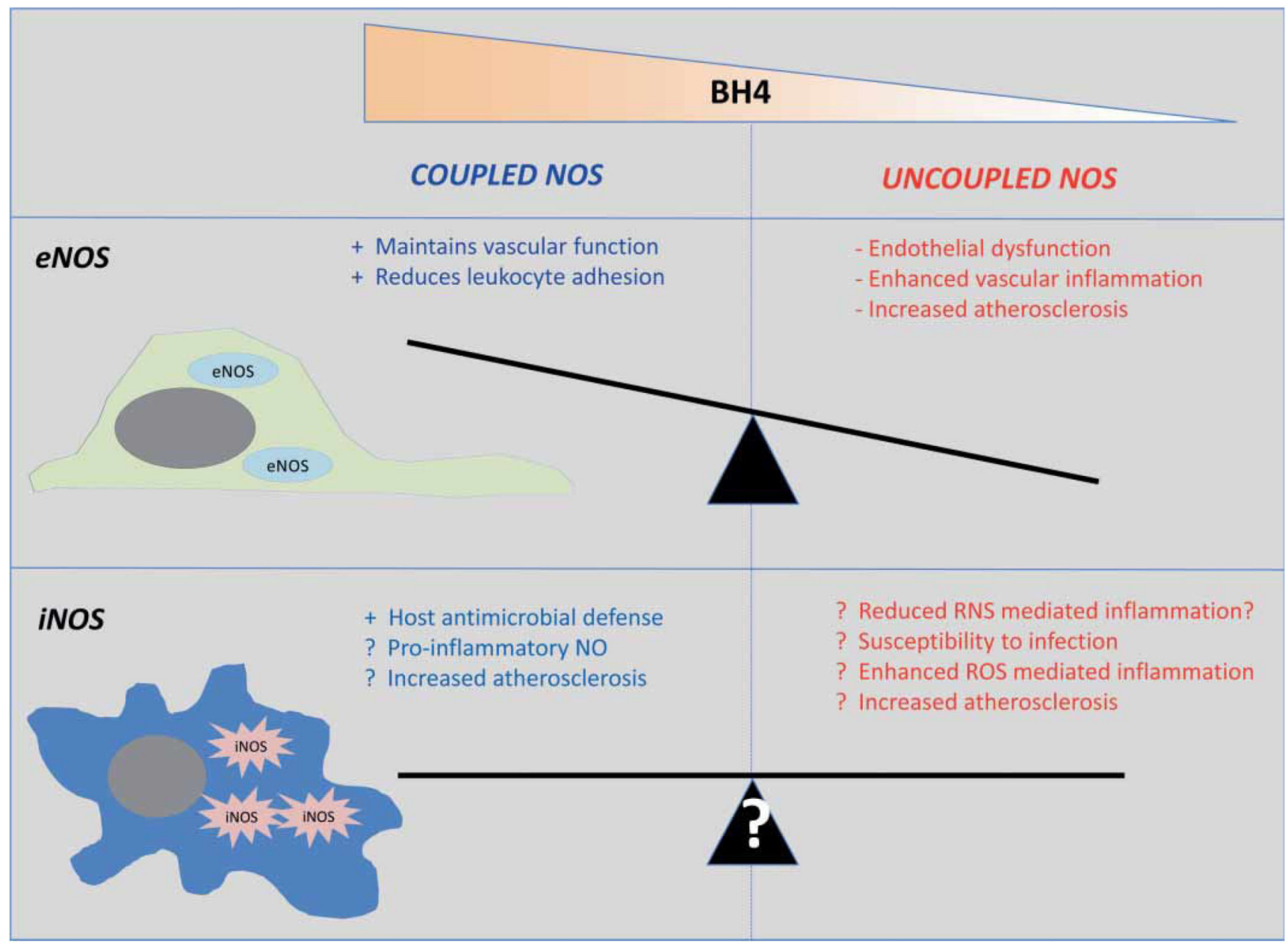

Figure 2. Whilst coupled and uncoupled endothelial cell eNOS has well defined roles and associations with vascular inflammation and disease, similar roles have not been assigned for iNOS.

iNOS expressed by leukocytes and other cells has the capacity to be deleterious in both its coupled and uncoupled state, but without defining the contribution of these different states of the enzyme it is hard to predict how therapeutic strategies aimed at modulating BH4 levels might impact on inflammatory vascular diseases. 


\section{Table 1}

Summary of key BH4 clinical studies.

\begin{tabular}{|c|c|c|c|c|}
\hline $1^{\text {st }}$ Author & Clinical setting & Intervention & Effect & Ref \\
\hline Stroes & Hypercholesterolaemia & $\begin{array}{l}\text { Acute infusion into the brachial } \\
\text { artery }\end{array}$ & $\begin{array}{l}\text { Restoration of endothelial function in the forearm in CAD } \\
\text { patients, no effect on controls }\end{array}$ & [74] \\
\hline Heitzer & Diabetes & $\begin{array}{l}\text { Acute infusion into the brachial } \\
\text { artery }\end{array}$ & $\begin{array}{l}\text { Improvement of forearm vasodilation in diabetic } \\
\text { individuals, no effect on controls. }\end{array}$ & [72] \\
\hline Higashi & Hypertension & $\begin{array}{l}\text { Acute infusion into the brachial } \\
\text { artery }\end{array}$ & $\begin{array}{l}\text { Restoration of forearm vasorelaxations to those of the } \\
\text { normal control subjects. }\end{array}$ & [73] \\
\hline Heitzer & Smokers & $\begin{array}{l}\text { Acute infusion into the brachial } \\
\text { artery }\end{array}$ & $\begin{array}{l}\text { Improvement of forearm blood flow in smokers, no effect } \\
\text { on non-smoker controls }\end{array}$ & [75] \\
\hline Maier & CAD patients & $\begin{array}{l}\text { Acute infusion into the } \\
\text { coronary artery }\end{array}$ & $\begin{array}{l}\text { Prevents Acetylcholine induced vasocontstriction in the } \\
\text { coronary artery in CAD patients. }\end{array}$ & [76] \\
\hline Cosentino & Hypercholesterolaemia & Oral treatment for 4 weeks & $\begin{array}{l}\text { Improved NO-mediated forearm vasodilatation to } \\
\text { acetylcholine, decreased plasma } \mathrm{F}_{2} \text {-isoprostanes } \\
\text { (oxidative stress biomarker). }\end{array}$ & [77] \\
\hline Cunnington & CAD patients & Oral treatment $2-6$ weeks & $\begin{array}{l}\text { No effect on in vivo or ex vivo (saphenous vein or } \\
\text { internal mammary artery) vascular function. }\end{array}$ & {$[78]$} \\
\hline Antoniades & CAD patients & $\begin{array}{l}\text { XX haplotype individuals } \\
\text { (unable to upregulate } \mathrm{BH} 4 \text { in } \\
\text { response to inflammation) }\end{array}$ & $\begin{array}{l}\text { XX individuals showed increased vascular superoxide } \\
\text { and reduced vasorelaxations to acetylcholine in ex vivo } \\
\text { saphenous vein or internal mammary artery segments. }\end{array}$ & [71] \\
\hline Antoniades & CAD patients & $\begin{array}{l}\text { XX haplotype individuals } \\
\text { (unable to upregulate BH4 in } \\
\text { response to inflammation) }\end{array}$ & $\begin{array}{l}\text { XX individuals showed no increase in plasma biopterins } \\
\text { in response to acute inflammation ( } S \text {. Typhi vaccination) } \\
\text { and showed a greater reduction in flow-mediated } \\
\text { dilatation in response to the inflammatory challenge. }\end{array}$ & {$[70]$} \\
\hline
\end{tabular}

